\title{
Longitudinal pathways of cerebrospinal fluid and positron emission tomography biomarkers of amyloid- $\beta$ positivity
}

\author{
Arianna Sala $\mathbb{1}^{1,2,3} \cdot$ Agneta Nordberg $\mathbb{(}^{1,4} \cdot$ Elena Rodriguez-Vieitez $\mathbb{D}^{1} \cdot$ for the Alzheimer's Disease Neuroimaging \\ Initiative
}

Received: 11 April 2020 / Revised: 9 October 2020 / Accepted: 2 November 2020 / Published online: 11 December 2020

(c) The Author(s) 2020. This article is published with open access

\begin{abstract}
Mismatch between CSF and PET amyloid- $\beta$ biomarkers occurs in up to $\approx 20 \%$ of preclinical/prodromal Alzheimer's disease individuals. Factors underlying mismatching results remain unclear. In this study we hypothesized that CSF/PET discordance provides unique biological/clinical information. To test this hypothesis, we investigated non-demented and demented participants with CSF amyloid- $\beta_{42}$ and $[18 \mathrm{~F}]$ Florbetapir PET assessments at baseline $(n=867)$ and at 2-year follow-up $(n=289)$. Longitudinal trajectories of amyloid- $\beta$ positivity were tracked simultaneously for CSF and PET biomarkers. In the longitudinal cohort $(n=289)$, we found that participants with normal CSF/PET amyloid- $\beta$ biomarkers progressed more frequently toward CSF/PET discordance than to full CSF/PET positivity $\left(\chi_{(1)}^{2}=5.40 ; p<0.05\right)$. Progression to $\mathrm{CSF}+/ \mathrm{PET}+$ status was ten times more frequent in cases with discordant biomarkers, as compared to csf-/pet- cases $\left(\chi_{(1)}^{2}=18.86 ; p<0.001\right)$. Compared to the CSF $+/$ pet - group, the csf-/PET + group had lower APOE$\varepsilon 4 \varepsilon 4$ prevalence $\left(\chi_{(6)}^{2}=197 ; p<0.001 ; n=867\right)$ and slower rate of brain amyloid- $\beta$ accumulation $\left(F_{(3,600)}=12.76 ; p<\right.$ $0.001 ; n=608)$. These results demonstrate that biomarker discordance is a typical stage in the natural history of amyloid- $\beta$ accumulation, with CSF or PET becoming abnormal first and not concurrently. Therefore, biomarker discordance allows for identification of individuals with elevated risk of progression toward fully abnormal amyloid- $\beta$ biomarkers, with subsequent risk of neurodegeneration and cognitive decline. Our results also suggest that there are two alternative pathways ("CSF-first" vs. "PET-first") toward established amyloid- $\beta$ pathology, characterized by different genetic profiles and rates of amyloid- $\beta$ accumulation. In conclusion, CSF and PET amyloid- $\beta$ biomarkers provide distinct information, with potential implications for their use as biomarkers in clinical trials.
\end{abstract}

Supplementary information The online version of this article (https:// doi.org/10.1038/s41380-020-00950-w) contains supplementary material, which is available to authorized users.

$\triangle$ Elena Rodriguez-Vieitez

elena.rodriguez-vieitez@ki.se

1 Division of Clinical Geriatrics, Center for Alzheimer Research, Department of Neurobiology, Care Sciences and Society, Karolinska Institutet, Stockholm, Sweden

2 Vita-Salute San Raffaele University, Milan, Italy

3 In Vivo Human Molecular and Structural Neuroimaging Unit, Division of Neuroscience, IRCCS San Raffaele Scientific Institute, Milan, Italy

4 Theme Aging, The Aging Brain, Karolinska University Hospital, Stockholm, Sweden

\section{Introduction}

The abnormal deposition of amyloid- $\beta$ into plaques is considered one of the earliest neuropathological events in Alzheimer's disease. Current research diagnostic criteria for Alzheimer's disease promote the use of either CSF (low levels of CSF amyloid- $\beta_{42}$ ) or PET imaging (high brain retention of amyloid- $\beta$ PET tracers) as equivalent measures of amyloid- $\beta$ pathology [1-4]. Amyloid- $\beta$ PET and CSF amyloid- $\beta_{42}$ usually provide highly concordant information, which has justified their interchangeable use in the diagnosis of Alzheimer's disease dementia [5, 6]. However, a significant proportion of cases showing discordant CSF and PET results has been consistently reported across multiple studies [6-34] (Supplementary Table 1), reaching up to $\approx 20 \%$ in prevalence [15] in preclinical/prodromal disease phases (e.g., $[13-15,18,19,28])$. To date, the interpretation of the CSF/ PET discordant findings is still unclear and the longitudinal 
evolution of the concordant and discordant CSF/PET groups remains unknown. While it has been suggested that discordance in amyloid- $\beta$ biomarkers might be related to measurement issues, due to different detection thresholds of CSF vs. PET techniques [13], recent studies have indicated that biomarker discordance might be biologically relevant, with each amyloid- $\beta$ biomarker providing distinct information $[15,19,35]$. There are several reasons to hypothesize that CSF and PET biomarkers measure different aspects of amyloid- $\beta$ pathology. CSF amyloid- $\beta_{42}$ is a marker of soluble amyloid- $\beta$, only indirectly related to fibrillar amyloid- $\beta$ in the brain [36]. Furthermore, CSF amyloid- $\beta_{42}$ can be affected by variations in amyloid precursor protein and amyloid- $\beta$ production, and by non-fibrillar aggregation [12]. On the other hand, amyloid- $\beta$ PET is a direct measure of fibrillar amyloid- $\beta$ deposition in the brain, as reported in antemortempostmortem correlative studies [37-40].

Hence, we hypothesize that discordance in amyloid- $\beta$ biomarkers provides unique information and that the existence of discordant groups may result from differences in amyloid- $\beta$ processing and kinetics in the CSF vs. in the brain. In particular, while CSF amyloid- $\beta_{42}$ represents an instantaneous measure of ongoing amyloid- $\beta$ accumulation, reflecting the availability of soluble amyloid- $\beta$ in the CSF, amyloid- $\beta$ PET represents an integral measure of previously accumulated amyloid- $\beta$, providing information on the resulting fibrillar amyloid- $\beta$ plaque accumulation. Thus, we expect the instantaneous CSF amyloid- $\beta_{42}$ concentration to be predictive of the subsequent rate of change in amyloid- $\beta$ PET standardized uptake value ratio (SUVr), but not vice-versa. To test this hypothesis, we aimed to: (a) test whether baseline CSF amyloid- $\beta_{42}$ levels significantly predict future rate of change in amyloid- $\beta$ PET SUVr; (b) test whether amyloid- $\beta$ PET SUVr at baseline does not significantly predict rate of change of CSF amyloid- $\beta_{42}$; (c) investigate the longitudinal evolution of CSF amyloid- $\beta_{42}$ and amyloid- $\beta$ PET biomarkers in a longitudinal cohort of participants stratified into four groups of concordant and discordant biomarkers (csf-/pet-, $\mathrm{CSF}+/$ pet-, csf-/PET+, and $\mathrm{CSF}+/ \mathrm{PET}+$ ).

\section{Participants and methods}

\section{Study design}

Data used in the preparation of this article were obtained from the Alzheimer's Disease Neuroimaging Initiative (ADNI) database (adni.loni.usc.edu). The ADNI was launched in 2003 as a public-private partnership, led by Principal Investigator Michael W. Weiner, MD. The primary goal of ADNI has been to test whether serial MRI, PET, other biological markers, and clinical and neuropsychological assessment can be combined to measure the progression of mild cognitive impairment and early Alzheimer's disease. In the current study, we included all ADNI participants with available concurrent CSF amyloid- $\beta_{42}$ and $[18 \mathrm{~F}]$ Florbetapir PET imaging measurements, obtained within a 3-month time interval. All participants gave written informed consent, as approved by local ethics committees and in accordance with the Declaration of Helsinki. For up-to-date information, see www.adni-info.org.

\section{Participants}

Data were downloaded from the ADNI database on October 24, 2018. Our cohort consisted of $n=867$ demented patients and non-demented participants, with available CSF amyloid- $\beta_{42}$ and [18F]Florbetapir PET imaging at baseline, obtained between April 2010 and April 2014 at 57 ADNI sites, within a time interval between CSF and PET measurements of 90 days $(\Delta t=0.08 \pm 17.18$ days $)$. A total of $n=608$ cases had a second [18F]Florbetapir PET scan performed after approximately 2 years $(\Delta t=2.01 \pm$ 0.68 years) and $n=348$ cases had a third scan after 4 years $(\Delta t=4.28 \pm 0.63$ years) from the baseline scan. A total of $n=305$ had a second CSF amyloid- $\beta_{42}$ measurement after $\sim 2$ years from baseline $(\Delta t=2 \pm 0.11$ years $)$.

\section{CSF amyloid- $\boldsymbol{\beta}_{42}$}

Following overnight fasting, CSF samples were acquired in the morning through lumbar puncture. CSF amyloid- $\beta_{42}$ measurement was performed based on the multiple xMAP Luminex platform (Luminex Corp) with INNO-BIA AlzBio3 immunoassay kit (Innogenetics) [41]. Longitudinal CSF samples belonging to the same participant were measured on the same plate. All data were derived from the UPENNBIOMK_MASTER.csv dataset.

\section{Amyloid- $\beta$ PET}

[18F]Florbetapir PET acquisition and analysis are thoroughly described elsewhere [13]. Following recommendations for longitudinal studies [42] we selected a composite reference region, including the whole cerebellum, brainstem/pons, and subcortical white matter, for SUVr estimation. A summary measure of global cortical uptake was calculated as the weighted-average uptake across regions of interest including frontal, anterior/posterior cingulate, lateral parietal, and lateral temporal lobes. All data were derived from the UCBERKELEYAV45_08_09_18.csv dataset.

\section{Other data}

Additional baseline and longitudinal measures of neurodegeneration, tau pathology, cognition, and neuropsychiatric 
symptomatology were available; genetic information was also retrieved. Number of participants with additional data available, at baseline and at follow-ups, is reported in the next paragraphs.

\section{FDG-PET}

FDG-PET acquisition and analysis are thoroughly described elsewhere [43]. A composite index for Alzheimer's diseaselike hypometabolism was available in $n=857$ participants ( $n=417$ at 2-year follow-up). Data were retrieved from the ADNIMERGE.csv dataset.

\section{Structural MRI}

T1-weighted MRI image acquisition and analysis are thoroughly described elsewhere [44]. Hippocampal, ventricles and whole-brain volume data were available in $n=799$ participants ( $n=614$ at 1 -year, $n=427$ at 1.5 -year, $n=335$ at 2-year follow-up). Volumes of target regions were corrected for total intracranial volume. Data were retrieved from ADNIMERGE.csv dataset.

\section{Other MRI measures}

Presence, localization and size of infarcts were evaluated by trained physicians on T1- and T2-weighted and FLAIR MRI images in $n=818$ participants [45]. Volume of white matter hyperintensities, indexing vascular brain injury, was quantified based on a Bayesian approach to segment highresolution T1 and FLAIR MRI images in $n=816$ participants [46]. Data were retrieved from the MRI_INFARCTS_11_16_15.csv and UCD_ADNI2_WMH_10_ 26_15.csv datasets.

\section{Other CSF measures}

CSF phosphorylated-tau ${ }_{181}(n=867$ at baseline; $n=307$ at 2-year follow-up) and total-tau ( $n=866$ at baseline; $n=$ 306 at 2-year follow-up) measurements were derived through AlzBio3 immunoassay. Data were retrieved from the UPENNBIOMK_MASTER.csv dataset. CSF amyloid$\beta_{40}$ measurements were obtained in $n=822$ participants at baseline through 2D-UPLC-tandem mass spectrometry. Data were retrieved from the UPENNMSMSABETA.csv and UPENNMSMSABETA2.csv datasets.

\section{Cognitive and neuropsychiatric measures}

We took into account the following scales for cognitive evaluation: Mini-Mental State Examination (MMSE) (available in $n=867$ participants, plus $n=711$ at $\sim 1$-year, $n=665$ at 2-year, $n=535$ at 3.5-year follow-up);
Alzheimer's disease Assessment Scale-cognitive subscale (ADAS- $\operatorname{cog} 11)(n=867 ; 724 ; 657 ; 528)$; Rey Auditory Verbal Learning Test (RAVLT), learning subscale $(n=$ $866 ; 722 ; 657 ; 520$ ).

Severity of depression and sleep disorders were evaluated by means of the Geriatric Depression Scale (GDS) $(n=865)$ and Neuropsychiatric Inventory, sleep subscale (NPI-K) $(n=860)$, respectively. Data were retrieved from the ADNIMERGE.csv, GDSSCALE.csv, and NPI.csv datasets.

\section{Genetic data}

$A P O E$ genotyping was performed using DNA extracted by Cogenics from a $3 \mathrm{ml}$ aliquot of whole blood $(n=867)$. We considered both the number of $A P O E-\varepsilon 4$ and $A P O E-\varepsilon 2$ alleles. Polygenic hazard score $(n=783)$ was computed based on a combination of $A P O E$ and 31 other genetic variants, as detailed elsewhere [47]. Data were retrieved from the APOE.csv and DESIKANLAB.csv datasets.

\section{Statistical analysis}

\section{Multivariable regression analyses}

We fitted linear, quadratic, and cubic regression models, testing whether baseline CSF amyloid- $\beta_{42}$ predicted the rate of change in [18F]Florbetapir PET SUVr (over 2-year and 4-year follow-up; $n=608$ and $n=348$ participants, respectively), and whether baseline [18F]Florbetapir PET SUVr predicted the rate of change in CSF amyloid- $\beta_{42}$ (over 2 -year follow-up; $n=305$ participants). CSF amyloid- $\beta_{42}$ and [18F]Florbetapir PET were included in the regression models as continuous variables. Sex, age, number of $A P O E$ $\varepsilon 4$ alleles and clinical group were entered as nuisance covariates. The assumptions of normal distribution and homoscedasticity of residuals, as well as the absence of multicollinearity between predictors, were tested and met in all regression models.

\section{Classification of participants}

Participants were classified as either csf-/pet-, $\mathrm{csf}-/ \mathrm{PET}+, \mathrm{CSF}+/$ pet - or $\mathrm{CSF}+/ \mathrm{PET}+$, based on cutoffs for abnormality of CSF amyloid- $\beta_{42}$ and [18F]Florbetapir PET biomarkers. CSF amyloid- $\beta_{42}$ measurements were classified as abnormal (CSF+) when $<192 \mathrm{pg} / \mathrm{ml}$. This cutoff was initially established to allow optimal discrimination between healthy controls and Alzheimer's disease cases confirmed at autopsy [48] and later validated using a diagnosis-free data-driven approach [49]. [18F] Florbetapir PET images were classified as abnormal (PET + ) when global cortical uptake was $>0.79$ SUVr [42]. 
This cutoff (optimized for longitudinal studies) was derived using linear regression from a well-validated cutoff for cross-sectional assessment (1.11 SUVr) [42], initially derived in healthy controls [50], then validated for discrimination between autopsy-confirmed healthy controls and Alzheimer's disease patients [51], and again using mixture model analysis [35].

\section{Longitudinal analyses}

To investigate the simultaneous longitudinal changes in CSF amyloid- $\beta_{42}$ and [18F]Florbetapir PET biomarkers, stratified by csf-/pet-, csf-/PET,$+ \mathrm{CSF}+/$ pet - or $\mathrm{CSF}+/ \mathrm{PET}+$ subgroups, we selected all participants $(n=$ 289 ) with CSF and PET amyloid- $\beta$ biomarkers available both at baseline and at the 2-year follow-up examinations, and obtained within 90 days. Differences in prevalence of longitudinal outcomes across subgroups were tested by means of $\chi^{2}$ tests.

\section{Group comparisons}

Group comparisons for continuous variables were performed via general linear modeling. Post-hoc tests were performed using Bonferroni correction for multiple comparisons. The assumptions of normality of residuals and homoscedasticity were tested; when not met, the estimates were bootstrapped with 1000 replicates, generating bias-corrected and accelerated 95\% confidence intervals. For categorical variables, $\chi^{2}$ tests were applied. Cox regression analysis was used for estimation of hazard risks. When the assumption of proportionality of hazards was not met, we run a stratified model. Sex, age, number of $A P O E-\varepsilon 4$ alleles, and clinical group were included as nuisance covariates.

All statistical analyses were performed in PASW Statistics 18 (SPSS Inc.), setting the significance level at $p<$ 0.05 (two tailed). Graphical renderings were performed in RStudio (v.1.1.456, http://www.rstudio.com/), using ggplot2 v.2.2.1.

\section{Results}

\section{Longitudinal changes in amyloid- $\beta$ CSF and PET measures in the whole cohort}

Table 1 shows baseline demographic and clinical characteristics of the participants, divided by clinical group $(n=867)$.

Table 1 Baseline descriptive statistics of the study cohort.

\begin{tabular}{|c|c|c|c|c|c|}
\hline & Healthy controls & $\begin{array}{l}\text { Subjective memory } \\
\text { complaints }\end{array}$ & Mild cognitive impairment & $\begin{array}{l}\text { Alzheimer's disease } \\
\text { dementia }\end{array}$ & $\begin{array}{l}\text { Test value; } \\
P \text { value }^{1}\end{array}$ \\
\hline$N(\%)$ & $185(21.34)$ & $90(10.38)$ & $445(51.33)$ & $147(16.96)$ & - \\
\hline Sex, $N(\%)$ male/female & $\begin{array}{l}89 / 96 \\
(48.11 / 51.89)\end{array}$ & $\begin{array}{l}36 / 54 \\
(40 / 60)\end{array}$ & $247 / 198(55.51 / 44.49)$ & $88 / 59(59.86 / 40.14)$ & $\begin{array}{l}\chi_{(3)}^{2}=11.79 ; \\
p<0.01\end{array}$ \\
\hline Age, years & $\begin{array}{l}74.74 \pm 6.70 \\
{[74.31 \pm 0.53]}\end{array}$ & $\begin{array}{l}72.15 \pm 5.42 \\
{[71.98 \pm 0.75]}\end{array}$ & $\begin{array}{l}72.06 \pm 7.41 \\
{[72.11 \pm 0.33]}\end{array}$ & $\begin{array}{l}74.66 \pm 7.29 \\
{[75.15 \pm 0.59]}\end{array}$ & $\begin{array}{l}F_{(3,861)}=9.67 \\
p<0.001^{\mathrm{a}}\end{array}$ \\
\hline Education, years & $\begin{array}{l}16.11 \pm 2.67 \\
{[16.16 \pm 0.20]}\end{array}$ & $\begin{array}{l}16.00 \pm 2.61 \\
{[16.05 \pm 0.28]}\end{array}$ & $\begin{array}{l}16.30 \pm 2.63 \\
{[16.30 \pm 0.13]}\end{array}$ & $\begin{array}{l}16.50 \pm 2.58 \\
{[16.41 \pm 0.22]}\end{array}$ & $\begin{array}{l}F_{(3,860)}=0.43 \\
p=0.73\end{array}$ \\
\hline MMSE & $\begin{array}{l}29.05 \pm 1.16 \\
{[29.02 \pm 0.09]}\end{array}$ & $\begin{array}{l}28.97 \pm 1.26 \\
{[28.85 \pm 0.13]} \\
29(28-30)\end{array}$ & $\begin{array}{l}28.03 \pm 1.76 \\
{[28.01 \pm 0.08]}\end{array}$ & $\begin{array}{l}22.99 \pm 2.49 \\
{[23.17 \pm 0.25]} \\
23(21-25)\end{array}$ & $\begin{array}{l}F_{(3,860)}=356.18 \\
p<0.001^{\mathrm{b}}\end{array}$ \\
\hline APOE- $\varepsilon 4$ alleles, $N(\%)(0 / 1 / 2)$ & $\begin{array}{l}138 / 42 / 5 \\
(74.60 / 22.70 / 2.70)\end{array}$ & $\begin{array}{l}61 / 28 / 1 \\
(67.78 / 31.11 / 1.11)\end{array}$ & $\begin{array}{l}229 / 171 / 45 \\
(51.46 / 38.43 / 10.11)\end{array}$ & $\begin{array}{l}51 / 67 / 29 \\
(34.69 / 45.58 / 19.73)\end{array}$ & $\begin{array}{l}\chi_{(6)}^{2}=74.68 \\
p<0.001\end{array}$ \\
\hline APOE- $\varepsilon 2$ alleles, $N(\%)(0 / 1 / 2)$ & $160 / 25 / 0(86.49 / 13.51 / 0)$ & 78/12/0 (86.67/13.33/0) & 409/36/0 (91.91/8.09/0) & $\begin{array}{l}140 / 6 / 1 \\
(95.24 / 4.08 / 0.68)\end{array}$ & $\begin{array}{l}\chi_{(6)}^{2}=16.11 \\
p<0.05\end{array}$ \\
\hline CSF amyloid- $\beta_{42}, \mathrm{pg} / \mathrm{ml}$ & $\begin{array}{l}199.32 \pm 51.88 \\
{[193.00 \pm 3.28]}\end{array}$ & $\begin{array}{l}202.7 \pm 48.5 \\
{[193.70 \pm 4.65]}\end{array}$ & $\begin{array}{l}174.55 \pm 52.32 \\
{[174.23 \pm 2.08]}\end{array}$ & $\begin{array}{l}138.85 \pm 40.06 \\
{[153.33 \pm 3.71]} \\
132(115-151)\end{array}$ & $\begin{array}{l}F_{(3,860)}=24.77 \\
p<0.001^{\mathrm{b}}\end{array}$ \\
\hline [18F]Florbetapir PET SUVr & $\begin{array}{l}0.80 \pm 0.11 \\
{[0.81 \pm 0.01]}\end{array}$ & $\begin{array}{l}0.82 \pm 0.12 \\
{[0.84 \pm 0.01]}\end{array}$ & $\begin{array}{l}0.88 \pm 0.14 \\
{[0.88 \pm 0.01]}\end{array}$ & $\begin{array}{l}1.01 \pm 0.14 \\
{[0.99 \pm 0.01]}\end{array}$ & $\begin{array}{l}F_{(3,860)}=56.82 \\
p<0.001^{\mathrm{b}}\end{array}$ \\
\hline
\end{tabular}

Results from GLM are corrected for sex, age, and number of APOE- $\varepsilon 4$ alleles. Data are reported as mean \pm standard deviation, unless indicated otherwise. Adjusted estimates of the mean and the respective standard error are reported in square brackets. Sex, age, number of APOE- 84 alleles, and clinical group were entered as nuisance covariates. For groups where variables are non-normally distributed, median (interquartile range) is also reported, in italics.

MMSE Mini-Mental State Examination, SUVr standardized uptake value ratio.

${ }^{1}$ Results of post-hoc comparisons, Bonferroni-corrected for multiple comparisons; significant differences:

${ }^{a}$ Healthy controls vs. mild cognitive impairment; subjective cognitive complaints vs. Alzheimer's disease dementia; mild cognitive impairment vs. Alzheimer's disease dementia.

${ }^{b}$ Healthy controls vs. mild cognitive impairment; healthy controls vs. Alzheimer's disease dementia; subjective cognitive complaints vs. mild cognitive impairment; subjective cognitive complaints vs. Alzheimer's disease dementia; mild cognitive impairment vs. Alzheimer's disease dementia. 


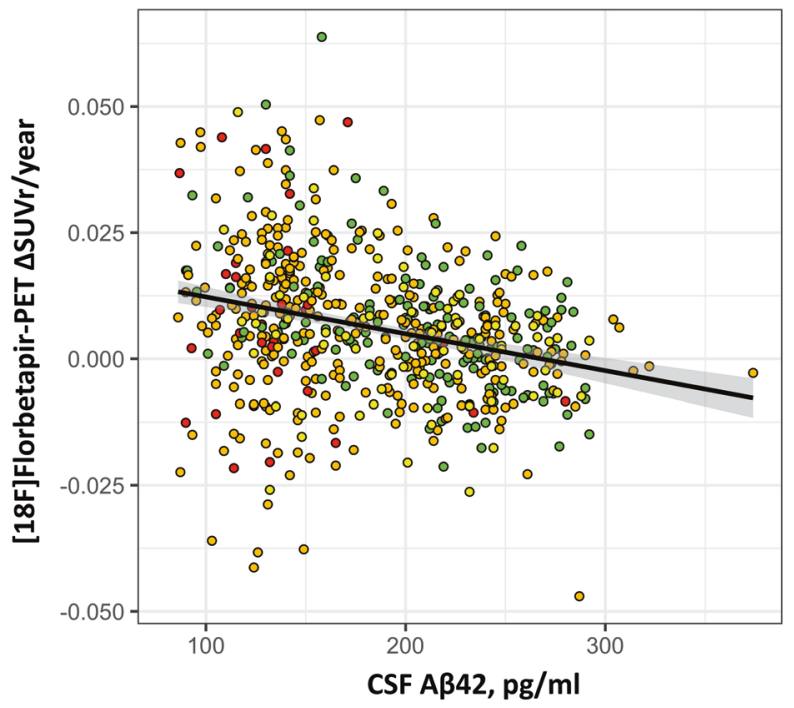

$\square$ HC $\square$ SMC

Fig. 1 Hypothesis validation-continuous measures. Scatter plots show the association between baseline CSF amyloid- $\beta_{42}$ vs. longitudinal changes in [18F]Florbetapir PET SUVr (left panel) and viceversa (right panel). Baseline CSF amyloid- $\beta_{42}$ significantly predicts the rate of change in $[18 \mathrm{~F}]$ Florbetapir PET SUVr, as computed on a 2-year time interval (left panel), the association being represented by a linear

Testing of our hypothesis by means of standard regression models in the whole cohort, considering participants with longitudinal amyloid- $\beta$ CSF $(n=305)$ and PET $(n=$ $608 ; n=348)$ measurements, confirmed both our predictions, i.e.,: (i) baseline CSF amyloid- $\beta_{42}$ values significantly predicted the rate of change in [18F]Florbetapir PET SUVr, as computed over a 2-year $\left(F_{(5,602)}=12.11, p<0.001 ; n=\right.$ 608 ; Fig. 1) and 4-year $\left(F_{(5,342)}=15.11, p<0.001 ; n=348\right.$; data not shown) time interval. Thus, CSF amyloid- $\beta_{42}$ levels linearly predicted $[18 \mathrm{~F}]$ Florbetapir PET accumulation, with lower CSF amyloid- $\beta_{42}$ levels associated with faster brain [18F]Florbetapir PET accumulation. Quadratic and cubic terms did not reach statistical significance in the respective models. (ii) Neither linear, quadratic nor cubic regression models significantly predicted the 2 -year rate of change in CSF amyloid- $\beta_{42}$ values as a function of baseline [18F] Florbetapir PET SUVr $\quad\left(F_{(4,302)}=1.06, \quad p=0.374\right.$; $F_{(5,301)}=0.92, \quad p=0.471 ; \quad F_{(6,300)}=0.95, \quad p=0.462$, respectively; $n=305$; Fig. 1 ).

\section{Longitudinal changes in amyloid- $\beta$ CSF and PET measures for each of the CSF/PET subgroups}

Table 2 shows baseline demographic and clinical characteristics of the participants, divided by CSF amyloid- $\beta_{42}$ and $[18$ F]Florbetapir PET biomarker profiles $(n=867)$.

The assessment of simultaneous 2-year longitudinal changes in both CSF amyloid- $\beta_{42}$ and [18F]Florbetapir PET

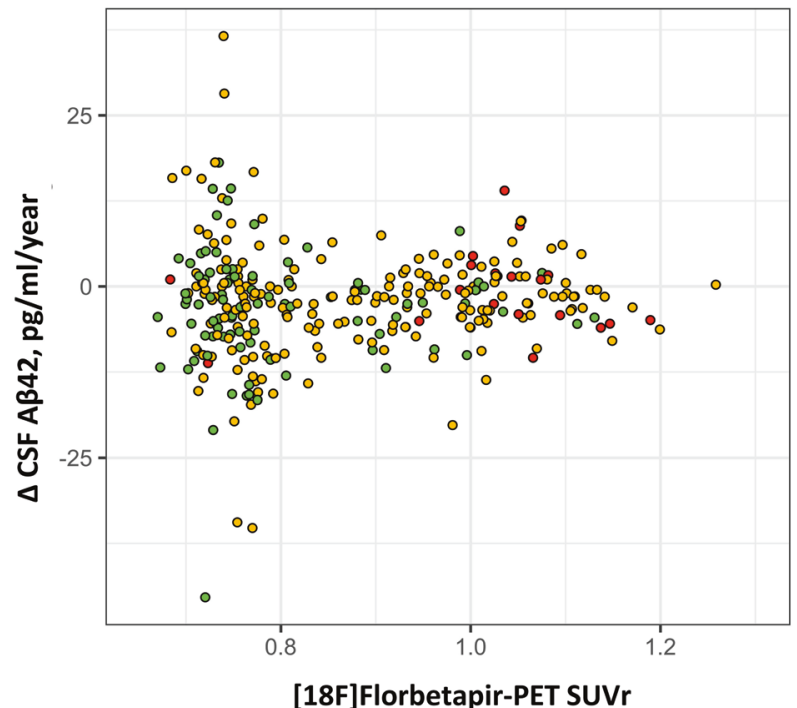

$\mathrm{MCl} \quad \square \mathrm{ADD}$

regression model. [18F]Florbetapir PET SUVr does not predict the rate of change in CSF amyloid- $\beta_{42}$, as computed on a 2 -year time interval (right panel). A $\beta 42$ amyloid- $\beta_{42}$, ADD Alzheimer's disease dementia, HC healthy control, MCI mild cognitive impairment, SMC subjective memory complaints, SUVr standardized uptake value ratio.

biomarkers in participants with both amyloid- $\beta$ CSF and PET measurements available, obtained within 90 days, both at baseline and at 2-year follow-up $(n=289$; Fig. 2), showed that participants with normal CSF amyloid- $\beta_{42}$ and [18F]Florbetapir PET biomarkers (csf-/pet- group) were more likely to progress to isolated CSF amyloid- $\beta_{42}$ positivity or to isolated [18F]Florbetapir PET positivity than to progress directly to full biomarker positivity (10.5\% [ $n=12 / 115]$ vs. $2.6 \%$ [3/115] of baseline csf-/pet- cases, $\left.\chi^{2}{ }_{(1)}=5.40 ; p<0.05\right)$. The frequency of progression to full biomarker positivity was significantly higher in both $\mathrm{CSF}+/$ pet - and csf-/PET + groups $(25.8 \%[n=8 / 31])$, compared to the csf-/pet- group $(2.6 \%[n=3 / 115])$ $\left(\chi_{(1)}^{2}=18.69 ; p<0.001 ; \chi_{(1)}^{2}=9.97 ; p<0.005\right.$, respectively), with no significant differences between $\mathrm{CSF}+/$ petand csf-/PET + groups $\left(\chi_{(1)}^{2}=0.26 ; p=0.613\right)$. Finally, $\mathrm{CSF}+/ \mathrm{PET}+$ participants were highly stable, with full biomarker positivity representing the endpoint of amyloid- $\beta$ biomarkers changes for $99.3 \%$ [ $n=142 / 143]$ of all cases.

At baseline $(n=867)$, when compared to the remaining groups, the groups with discordant amyloid- $\beta$ biomarkers presented with "intermediate" levels of the pathological amyloid- $\beta$ biomarker, i.e., (i) the $\mathrm{CSF}+/$ pet - group had significantly lower CSF amyloid- $\beta_{42}$ levels than those with normal CSF amyloid- $\beta_{42}$ values (both $\mathrm{csf}-/$ pet- and csf-/PET + ), but significantly higher CSF amyloid- $\beta_{42}$ levels as compared to the $\mathrm{CSF}+/ \mathrm{PET}+$ group; (ii) the csf-/PET + group had significantly higher [18F]Florbetapir 
Table 2 Baseline descriptive statistics for concordant and discordant biomarker groups.

\begin{tabular}{|c|c|c|c|c|c|}
\hline & csf-/pet- & $\operatorname{csf}-/ \mathrm{PET}+$ & $\mathrm{CSF}+/$ pet - & $\mathrm{CSF}+/ \mathrm{PET}+$ & $\begin{array}{l}\text { Test value; } p \\
\text { value }^{1}\end{array}$ \\
\hline$N(\%)$ & $300(34.60)$ & $44(5.07)$ & $62(7.15)$ & $461(53.17)$ & - \\
\hline $\begin{array}{l}\text { Clinical group, } N(\%) \text { (healthy } \\
\text { controls/subjective memory } \\
\text { complaints/mild cognitive } \\
\text { impairment/Alzheimer's disease } \\
\text { dementia) }\end{array}$ & $\begin{array}{l}99 / 43 / 145 / 13 \\
(33 / 14.33 / 48.33 / 4.33)\end{array}$ & $\begin{array}{l}9 / 12 / 22 / 1 \\
(20.45 / 27.27 / 50 / 2.27)\end{array}$ & $\begin{array}{l}23 / 6 / 28 / 5 \\
(37.10 / 9.68 / 45.16 / 8.06)\end{array}$ & $\begin{array}{l}54 / 29 / 250 / 128 \\
(11.71 / 6.29 / 54.23 / 27.77)\end{array}$ & $\begin{array}{l}\chi_{(9)}^{2}=140.64 ; \\
p<0.001\end{array}$ \\
\hline Sex, $N(\%)$ (male/female) & $\begin{array}{l}164 / 136 \\
(54.67 / 45.33)\end{array}$ & $\begin{array}{l}14 / 30 \\
(31.82 / 68.18)\end{array}$ & $\begin{array}{l}42 / 20 \\
(67.74 / 32.26)\end{array}$ & $\begin{array}{l}240 / 221 \\
(52.06 / 47.94)\end{array}$ & $\begin{array}{l}\chi_{(3)}^{2}=13.83 \\
p<0.005\end{array}$ \\
\hline Age, years & $\begin{array}{l}72.00 \pm 7.36 \\
{[70.41 \pm 0.44]}\end{array}$ & $\begin{array}{l}70.25 \pm 6.35 \\
{[69.67 \pm 0.91]}\end{array}$ & $\begin{array}{l}74.22 \pm 7.84 \\
{[74.31 \pm 1.13]}\end{array}$ & $\begin{array}{l}73.90 \pm 7.10 \\
{[75.19 \pm 0.37]}\end{array}$ & $\begin{array}{l}F_{(3,860)}=21.04 \\
p<0.001^{\mathrm{a}}\end{array}$ \\
\hline Education, years & $\begin{array}{l}16.21 \pm 2.63 \\
{[16.37 \pm 0.17]}\end{array}$ & $\begin{array}{l}16.11 \pm 2.53 \\
{[16.23 \pm 0.40]}\end{array}$ & $\begin{array}{l}15.82 \pm 2.65 \\
{[15.87 \pm 0.34]}\end{array}$ & $\begin{array}{l}16.37 \pm 2.63 \\
{[16.25 \pm 0.14]}\end{array}$ & $\begin{array}{l}F_{(3,859)}=0.6 \\
p=0.613\end{array}$ \\
\hline MMSE & $\begin{array}{l}28.57 \pm 1.70 \\
{[27.79 \pm 0.12]} \\
29(28-30)\end{array}$ & $\begin{array}{l}28.64 \pm 1.82 \\
{[27.94 \pm 0.29]} \\
29(28-30)\end{array}$ & $\begin{array}{l}28.18 \pm 2.18 \\
{[27.66 \pm 0.25]}\end{array}$ & $\begin{array}{l}26.59 \pm 3.06 \\
{[27.23 \pm 0.12]}\end{array}$ & $\begin{array}{l}F_{(3,859)}=3.51 \\
p<0.05^{\mathrm{b}}\end{array}$ \\
\hline APOE- $\varepsilon 4$ alleles, $N(\%)(0 / 1 / 2)$ & $\begin{array}{l}251 / 49 / 0 \\
(83.67 / 16.33 / 0)\end{array}$ & $\begin{array}{l}30 / 14 / 0 \\
(68.18 / 31.82 / 0)\end{array}$ & $\begin{array}{l}40 / 15 / 7 \\
(64.52 / 24.19 / 11.29)\end{array}$ & $\begin{array}{l}158 / 230 / 73 \\
(34.27 / 49.89 / 15.84)\end{array}$ & $\begin{array}{l}\chi_{(6)}^{2}=197 \\
p<0.001\end{array}$ \\
\hline APOE- $\varepsilon 2$ alleles, $N(\%)(0 / 1 / 2)$ & $\begin{array}{l}254 / 46 / 0 \\
(84.67 / 15.33 / 0)\end{array}$ & $38 / 6 / 0(86.36 / 13.64 / 0)$ & $\begin{array}{l}55 / 7 / 0 \\
(88.71 / 11.29 / 0)\end{array}$ & $\begin{array}{l}440 / 20 / 1 \\
(95.44 / 4.34 / 0.22)\end{array}$ & $\begin{array}{l}\chi_{(6)}^{2}=29.93 \\
p<0.001\end{array}$ \\
\hline CSF amyloid- $\beta_{42}, \mathrm{pg} / \mathrm{ml}$ & $\begin{array}{l}235.41 \pm 27.94 \\
{[230.18 \pm 1.58]}\end{array}$ & $\begin{array}{l}231.57 \pm 28.83 \\
{[227.03 \pm 3.77]}\end{array}$ & $\begin{array}{l}161.94 \pm 23.80 \\
{[160.91 \pm 3.16]}\end{array}$ & $\begin{array}{l}135.26 \pm 23.69 \\
{[139.24 \pm 1.26]}\end{array}$ & $\begin{array}{l}F_{(3,859)}=625.34 ; \\
p<0.001^{\mathrm{c}}\end{array}$ \\
\hline [18F]Florbetapir PET SUVr & $\begin{array}{l}0.73 \pm 0.03 \\
{[0.75 \pm 2.9 \mathrm{E}-03]}\end{array}$ & $\begin{array}{l}0.82 \pm 0.03 \\
{[0.83 \pm 5.88 \mathrm{E}-03]} \\
0.81(0.80-0.83)\end{array}$ & $\begin{array}{l}0.75 \pm 0.03 \\
{[0.76 \pm 4.41 \mathrm{E}-03]}\end{array}$ & $\begin{array}{l}1 \pm 0.1 \\
{[0.99 \pm 5 E-03]}\end{array}$ & $\begin{array}{l}F_{(3,859)}=487.61 \\
p<0.001^{\mathrm{d}}\end{array}$ \\
\hline
\end{tabular}

Results from GLM are corrected for sex, age, diagnostic group and number of APOE- $\varepsilon 4$ alleles. Data are reported as mean \pm standard deviation, unless indicated otherwise. Adjusted estimates of the mean and the respective standard error are reported in square brackets. Sex, age, number of APOE- $\varepsilon 4$ alleles and clinical group were entered as nuisance covariates. For groups where variables are non-normally distributed, median (interquartile range) is also reported, in italics.

MMSE Mini-Mental State Examination, SUVr standardized uptake value ratio.

${ }^{1}$ Significant differences after post-hoc comparisons, at $p<0.05$, Bonferroni-corrected for multiple comparisons:

${ }^{\mathrm{a}}$ csf-/pet- vs. CSF+/pet-; csf-/pet- vs. CSF+/PET +; csf-/PET+ vs. CSF+/pet-; csf-/PET + vs. CSF+/PET + .

${ }^{\mathrm{b}} \mathrm{csf}-/$ pet - vs. CSF+/PET + .

${ }^{c}$ csf-/pet- vs. CSF+/pet-; csf-/pet- vs. CSF+/PET+; csf-/PET+ vs. CSF+/pet-; csf-/PET+ vs. CSF+/PET+; CSF+/pet - vs. CSF+/PET+. ${ }^{\mathrm{d}} \mathrm{csf}-/$ pet - vs. csf-/PET + ; csf-/pet- vs. $\mathrm{CSF}+/ \mathrm{PET}+; \mathrm{csf}-/ \mathrm{PET}+$ vs. $\mathrm{CSF}+/$ pet-; csf-/PET + vs. $\mathrm{CSF}+/ \mathrm{PET}+; \mathrm{CSF}+/ \mathrm{pet}-\mathrm{vs} . \mathrm{CSF}+/ \mathrm{PET}+$.

PET retention compared to either the csf-/pet- or $\mathrm{CSF}+$ /pet - groups but significantly lower as compared to the CSF+/PET+ level (Table 2). This pattern was consistently observed at the regional level as well (Supplementary Fig. 1; Supplementary Table 2). We also observed a dissociation between baseline [18F]Florbetapir PET retention and the rate of [18F]Florbetapir PET accumulation, where rates of [18F]Florbetapir PET accumulation are expressed in $\Delta \mathrm{SUVr} /$ year, as computed over 2-year $(n=608)$ and 4-year $(n=348)$ follow-up time intervals. Significant [18F]Florbetapir PET accumulation rates were reported in CSF+/PET + [95\% CI at $2 \mathrm{y}: 0.009-0.012 ; 4 \mathrm{y}$ : $0.011-0.013$ ] and $\mathrm{CSF}+/$ pet - cases [95\% CI at $2 \mathrm{y}$ : $0.003-0.009 ; 4$ y: 0.004-0.009], while minimal to null accumulation was present in csf-/pet- $[95 \% \mathrm{CI}$ at $2 \mathrm{y}$ : $0.001-0.002$; 4 y: 0-0.002] and csf-/PET $+[95 \% \mathrm{CI}$ at $2 \mathrm{y}$ : 0-0.003; 4 y: 0-0.005] cases (Supplementary Fig. 1; Supplementary Table 2 ).

When systematically comparing discordant and concordant groups considering (i) biomarkers for tau pathology and neurodegeneration, (ii) clinical and neuropsychological measures, (iii) genetic assessments and (iv) confounding factors (data available in up to $n=867$ participants; see "Participants and Methods" for the precise values), we found that:

(i) A systematic comparison of biomarkers for tau pathology and neurodegeneration among CSF/PET groups revealed no significant differences between the $\mathrm{CSF}+/$ petand csf-/PET + groups. Only the $\mathrm{CSF}+/ \mathrm{PET}+$ group presented with increased baseline tau pathology (as measured by CSF phosphorylated-tau ${ }_{181}$ ) and neurodegeneration (as measured by CSF total-tau, [18F] Fluorodeoxyglucose PET, and MRI), and faster rate of neurodegeneration as compared to the csf-/pet- group (Supplementary Table 3).

(ii) Groups with discordant biomarkers were not at increased risk of clinical progression (csf-/PET+: hazard risk $=1.14$ [95\% CI: 0.44-2.99], $p=0.789$; CSF+/pet - : hazard risk $=1.59$ [95\% CI: $0.74-3.42], p=0.235$ ) as compared to the csf-/pet- group, and in contrast with the $\mathrm{CSF}+/ \mathrm{PET}+$ group (hazard risk $=4.12 \quad[95 \%$ CI: 2.59-6.56], $p<0.001)$. Discordant groups presented no difference in either baseline levels or rate of cognitive decline as measured by MMSE, ADAS- $\operatorname{cog} 11$ and 


\section{csf-/pet-}

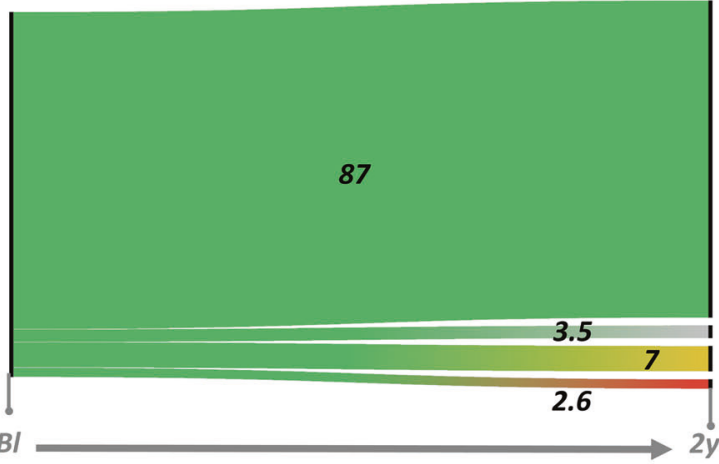

\section{CSF+/pet-}

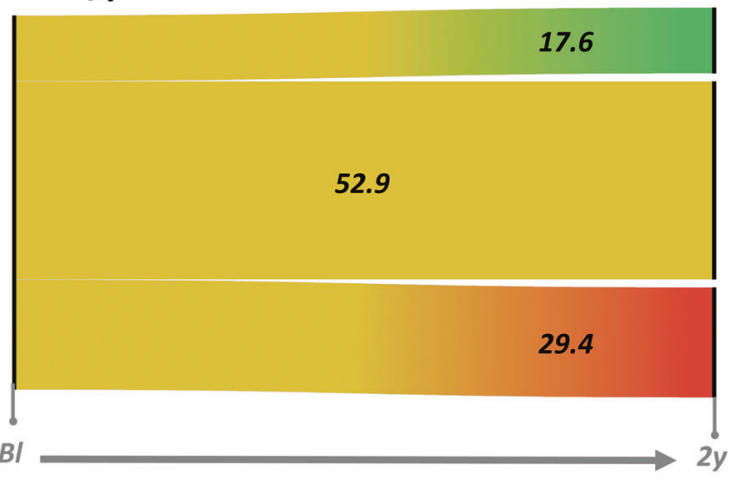

csf-/pet-

$\square$ csf-/PET+

Fig. 2 Trajectories of biomarker changes across cases with concordant and discordant amyloid- $\beta$ biomarkers. Alluvial plots show trajectories of biomarker changes in csf-/pet-, csf-/PET + ,

RAVLT-learning subscale, when compared to the csf-/pet- group. Only the CSF+/PET + group presented with significant rates of cognitive decline compared to all other groups (Supplementary Table 4). When examining the severity of neuropsychiatric depressive symptoms, we found that the GDS score was significantly higher in the csf-/PET+ group (2.09 [2.07]), as compared to all remaining groups (csf-/pet-: 1.48 [1.66]; $\mathrm{CSF}+/$ pet-: 1.50 [1.56]; CSF+/PET+: 1.44 [1.36] $) \quad\left(F_{(3,857)}=2.75\right.$, $p<0.05$ ) (Supplementary Table 4).

(iii) Genetic assessment revealed a significant association between number of APOE- $\varepsilon 4$ alleles and CSF/PET amyloid- $\beta$ biomarkers profile $\left(\chi_{(6)}^{2}=197 ; \quad p<0.001\right.$; Table 2). Although most of the association was driven by fully negative vs. fully positive cases, csf-/PET + cases presented with a lower prevalence of $A P O E-\varepsilon 4 \varepsilon 4$ genotype than expected $(0 \%$; standardized residual $=-2)$. Isolated PET positivity was associated with female sex (standardized residual $=2.1 ; \chi_{(3)}^{2}=13.83 ; p<0.005$; Table 2 ).

(iv) When examining a series of confounding factors in amyloid- $\beta$ fluid and molecular imaging assessments

\section{csf-/PET+}

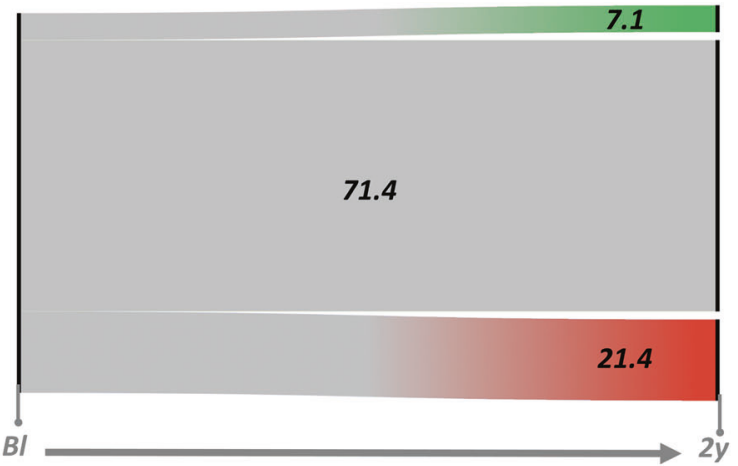

\section{CSF+/PET+}

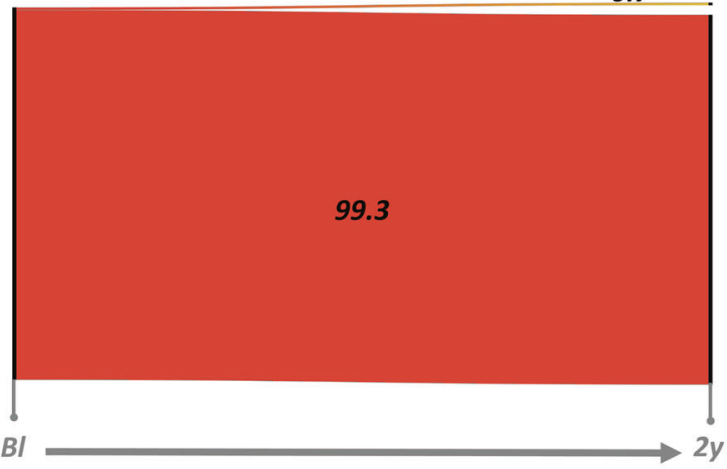

\section{CSF+/pet- $\square \mathrm{CSF}+/ \mathrm{PET}+$}

$\mathrm{CSF}+/$ pet - and $\mathrm{CSF}+/ \mathrm{PET}+$ cases (from top left corner, clockwise). Two time points are displayed for each group, i.e., baseline and 2-year follow-up. bl baseline, 2y 2-year follow-up.

(Supplementary Table 5), we found no differences across concordant and discordant CSF/PET groups, namely: (a) size of ventricles, as a proxy for disrupted CSF clearance mechanisms [52] was not disproportionally affected in cases with discordant amyloid- $\beta$ biomarkers compared to $\mathrm{CSF}+/ \mathrm{PET}+$ cases $(\mathrm{csf}-/ \mathrm{PET}+: 0.02 \pm 0.02 ; \mathrm{CSF}+/$ pet- : $0.03 \pm 0.02 ; \mathrm{CSF}+/ \mathrm{PET}+: 0.03 \pm 0.01$ ); (b) no significant difference was reported for frequency/severity of sleep disturbances, previously associated with CSF amyloid- $\beta_{42}$ changes [53] $\left(F_{(3,852)}=0.26, p=0.854\right)$; (c) time interval between [18F]Florbetapir injection and start of image acquisition was not increased in cases with discordant amyloid- $\beta$ biomarkers $\quad\left(F_{(3,857)}=1.40, \quad p=0.242\right)$; (d) occurrence of infarcts $\left(\chi_{(3)}^{2}=2.69, \quad p=0.442\right)$ as well as volume of white matter hyperintensities $\left(F_{(3,808)}=\right.$ $0.57, p=0.634$ ) within the SUVr reference region did not differ between groups. Finally, lack of significantly decreased CSF amyloid- $\beta_{40}$ levels in csf-/ PET + individuals (csf-/pet-: $8718.85 \mathrm{pg} / \mathrm{ml}$; csf-/PET+: $9788.26 \mathrm{pg} / \mathrm{ml}$ ), together with presence of relevant, but not severe, levels of occipital amyloid- $\beta$ retention 
Fig. 3 Trajectories toward full amyloid- $\beta$ biomarkers

abnormality. The figure shows the different trajectories toward full abnormality in amyloid- $\beta$ biomarkers. Different possible pathways, i.e., either CSF amyloid- $\beta_{42}$ positivity first or [18F]Florbetapir PET positivity first, are possible.

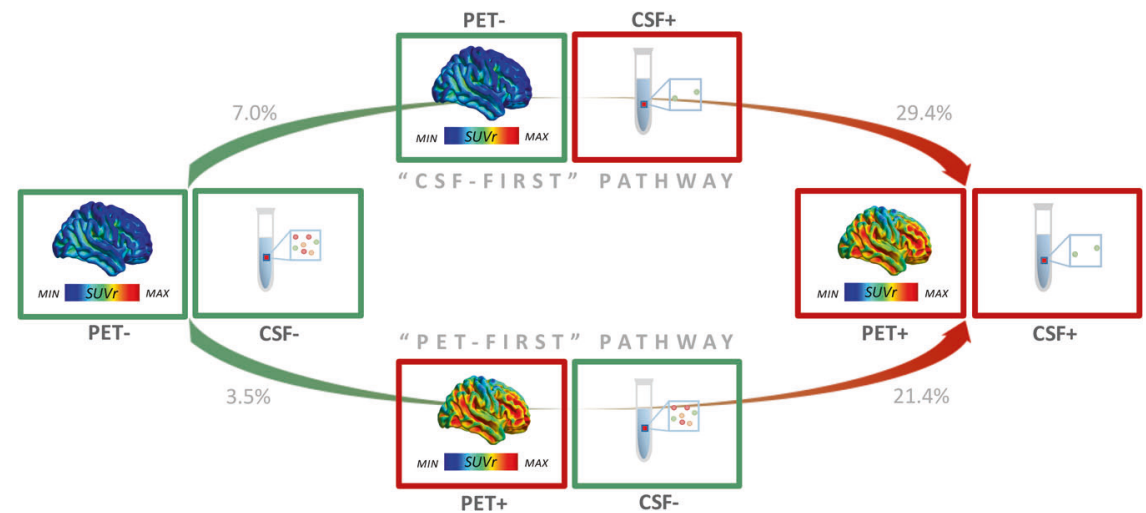

(csf-/pet-: 0.75 [0.06]; csf-/PET+: 0.83 [0.06]; CSF+/ PET +: 0.94 [0.13]) makes it unlikely that csf-/PET + cases are due to cerebral amyloid angiopathy.

\section{Discussion}

Discordance in CSF and PET measures of amyloidosis is fairly common, occurring in $\approx 20 \%$ of individuals at preclinical and prodromal stages of Alzheimer's disease [15]. Yet, guidelines on how to correctly interpret discordant CSF/PET results are lacking. In this study, we validated the hypothesis that discordance in CSF/PET amyloid- $\beta$ biomarkers provides unique information and that the existence of discordant groups may result from differences in amyloid- $\beta$ processing and kinetics in the CSF vs. in the brain. Through systematic assessment of discordant amyloid- $\beta$ biomarker results at both crosssectional and longitudinal levels, we showed that biomarker discordance represents a natural phase in the progression of amyloid- $\beta$ pathology, with either CSF or PET amyloid- $\beta$ biomarkers becoming abnormal first and not concurrently. We subsequently identified two mutually exclusive trajectories ("CSF-first" vs. "PETfirst") toward established amyloid- $\beta$ pathology, characterized by different genetic profiles and rates of amyloid- $\beta$ accumulation ("fast" vs. "slow" accumulators, respectively). In addition, we showed that biomarker discordance was not due to systematic errors in CSF and PET measurements. Instead, biomarker discordance carried prognostic relevance as a marker of progression toward established amyloid- $\beta$ pathology, thus allowing identification of individuals at subsequent risk of clinical progression, cognitive decline, increased neurodegeneration, and tau pathology.

First, for the longitudinal cohort as a whole, decreased CSF amyloid- $\beta_{42}$ levels at baseline were predictive of subsequent increases in amyloid- $\beta$ plaques in the brain as measured by longitudinal [18F]Florbetapir PET (Fig. 1). This observation supports the concept that decreased CSF amyloid- $\beta_{42}$ might be a proxy for the rate at which soluble amyloid- $\beta$ isoforms are being trapped into nonsoluble amyloid- $\beta$ aggregates in the brain as measured by PET. Consistent with this observation, and in participants with normal CSF/PET amyloid- $\beta$ biomarkers, CSF amyloid- $\beta_{42}$, and [18F]Florbetapir PET levels did not become pathological at the same time. Preferentially, CSF amyloid- $\beta_{42}$ became abnormal first without detectable brain [18F]Florbetapir PET uptake (7\% of csf-/pet - cases progressed to $\mathrm{CSF}+/$ pet-). This evidence, coupled with the finding that about $30 \%$ of $\mathrm{CSF}+$ /pet - cases progressed to $\mathrm{CSF}+/ \mathrm{PET}+$, qualifies isolated CSF positivity as a preferential pathway from fully normal to fully pathological amyloid- $\beta$ biomarkers, consistent with previous reports $[19,24]$.

However, our cross-sectional and longitudinal analyses in this large cohort provided evidence for the existence of an additional alternative pathway, where [18F]Florbetapir PET became abnormal first. Firstly, and consistent with previous reports [6-8, 13-15, 17, 18, 20-32, 34] (Supplementary Table 1), we observed isolated [18F]Florbetapir PET positivity in a non-negligible percentage of cases. This cross-sectional evidence alone questions whether isolated CSF amyloid- $\beta_{42}$ positivity represents the only possible pathway toward fully abnormal amyloid- $\beta$ biomarkers. Indeed, our longitudinal results showed that-in a proportion of participants with normal amyloid- $\beta$ biomarkers at baseline- $[18 \mathrm{~F}]$ Florbetapir PET became abnormal first, while levels of CSF amyloid- $\beta_{42}$ were still normal (3.5\% of csf-/pet- cases progressed to csf-/PET + ). In addition, about $20 \%$ of the participants with isolated pathological $[18 \mathrm{~F}]$ Florbetapir PET progressed to $\mathrm{CSF}+/ \mathrm{PET}+$. These longitudinal data suggest that cases with isolated [18F] Florbetapir PET positivity are part of an alternative 
(PET-first) pathway toward established amyloid- $\beta$ pathology (Fig. 3).

Of note, the frequency of progression from csf-/pet- to either $\mathrm{CSF}+/$ pet - or PET+/csf - was not significantly different; this finding supports the concept that both CSFfirst and PET-first pathways are relevant. Furthermore, our study found a slower rate of $[18 \mathrm{~F}]$ Florbetapir PET accumulation in the csf-/PET + compared to the CSF+/petgroup. Thus, the existence of cases with discordant biomarkers could be partly explained by differences in amyloid- $\beta$ accumulation dynamics, with csf-/PET + and $\mathrm{CSF}+/$ pet - cases representing "slow" and "fast" amyloid- $\beta$ accumulators, respectively. This explanation is also supported by the lower prevalence of $A P O E-\varepsilon 4 \varepsilon 4$ genotype among csf-/PET + individuals found in this study (Table 2), considering that a slower rate of amyloid- $\beta$ plaque accumulation is found in non- $A P O E-\varepsilon 4$ carriers compared to $A P O E-\varepsilon 4$ carriers [54]. In contrast, $\mathrm{CSF}+/$ petcases, despite presenting no evidence of significant amyloid- $\beta$ PET tracer retention, accumulated plaques in the brain at a relatively fast rate, a finding consistent with the higher prevalence of $A P O E-\varepsilon 4 \varepsilon 4$ genotype found in the $\mathrm{CSF}+/$ pet - group compared to that in the csf-/PET+ group (Table 2).

On a technical note, it has been argued that occurrence of discordant findings might be inherently dependent on the choice of cutoffs to establish biomarkers' abnormality [36]. Still, independently of the type of cutoffs adopted, previous studies consistently reported a proportion of discordant amyloid- $\beta$ biomarker results, even when using cutoffs specifically designed to optimize agreement between biomarkers [14, 16-18, 20, 21, 23, 25-27, 29-31] (Supplementary Table 1). Similarly, it has been proposed that discordant cases might be due to false negative or false positive results, CSF analytical factors or failed PET scans [19]. Our assessment of a comprehensive series of confounding factors in this study argues against this explanation. Altogether, our results argue against the claim that discordant findings in amyloid- $\beta$ biomarkers systematically represent false positive/negative results, suggesting instead that amyloid- $\beta$ biomarkers discordance, per se, carries relevant information with respect to brain amyloid- $\beta$ burden and accumulation rate. Most importantly, amyloid- $\beta$ biomarkers discordance represents a strong predictor of future progression toward full amyloid- $\beta$ biomarkers abnormalities (Fig. 2), thus allowing prompt identification of individuals that will be at higher subsequent risk for clinical progression and cognitive decline, steeper rates of neurodegeneration, and tau pathology [14, 15, 19, 28].

This study has some limitations. We acknowledge that the sample size of discordant biomarker groups was relatively limited ( $n=62$ for CSF + /pet - and $n=44$ for csf-/ PET + cases). Although we used all longitudinal CSF/PET data available from ADNI at the time of our study, joint CSF/PET examinations were available at two longitudinal time points only, thus future studies incorporating additional longitudinal CSF/PET time points will be valuable to confirm our findings.

In conclusion, biomarker discordance allows for identification of individuals with elevated risk of progression toward fully abnormal amyloid- $\beta$ biomarkers, with subsequent risk of neurodegeneration and cognitive decline. Our results also suggest that there are two alternative pathways ("CSF-first" vs. "PET-first") toward established amyloid- $\beta$ pathology, characterized by different genetic profiles and rates of amyloid- $\beta$ accumulation. In conclusion, CSF and PET amyloid- $\beta$ biomarkers provide complementary rather than redundant information, with potential implications for their use as biomarkers in clinical trials.

Acknowledgements The project leading to this article has received funding from the Innovative Medicines Initiative 2 Joint Undertaking under grant agreement no. 115952. This Joint Undertaking receives the support from the European Union's Horizon 2020 research and innovation program and European Federation of Pharmaceutical Industries and Associations (EFPIA). This work is also supported by the Swedish Alzheimer Foundation (Alzheimerfonden), Swedish Brain Foundation (Hjärnfonden), Swedish Dementia Association (Demensfonden), Swedish Foundation for Strategic Research (SSF) [project RB13-0192], Swedish Research Council [projects K2014-61X-05817, 2017-02965, 2017-06086], Åke Wiberg Foundation, and the Stockholm County Council-Karolinska Institutet regional agreement on medical training and clinical research (ALF grant). Funding sources had no role in study design, collection, analysis and interpretation of data, writing of the report or decision to submit the article for publication. We gratefully acknowledge all research participants for their time and dedication to these studies. Data collection and sharing for this project was funded by the ADNI (National Institutes of Health Grant U01 AG024904) and DOD ADNI (Department of Defense award number W81XWH-12-2-0012). ADNI is funded by the National Institute on Aging, the National Institute of Biomedical Imaging and Bioengineering, and through generous contributions from the following: AbbVie, Alzheimer's Association; Alzheimer's Drug Discovery Foundation; Araclon Biotech; BioClinica, Inc.; Biogen; Bristol-Myers Squibb Company; CereSpir, Inc.; Cogstate; Eisai Inc.; Elan Pharmaceuticals, Inc.; Eli Lilly and Company; EuroImmun; F. Hoffmann-La Roche Ltd and its affiliated company Genentech, Inc.; Fujirebio; GE Healthcare; IXICO Ltd.; Janssen Alzheimer Immunotherapy Research \& Development, LLC.; Johnson \& Johnson Pharmaceutical Research \& Development LLC.; Lumosity; Lundbeck; Merck \& Co., Inc.; Meso Scale Diagnostics, LLC.; NeuroRx Research; Neurotrack Technologies; Novartis Pharmaceuticals Corporation; Pfizer Inc.; Piramal Imaging; Servier; Takeda Pharmaceutical Company; and Transition Therapeutics. The Canadian Institutes of Health Research is providing funds to support ADNI clinical sites in Canada. Private sector contributions are facilitated by the Foundation for the National Institutes of Health (www.fnih.org). The grantee organization is the Northern California Institute for Research and Education, and the study is coordinated by the Alzheimer's Therapeutic Research Institute at the University of Southern California. ADNI data are disseminated by the Laboratory for Neuro Imaging at the University of Southern California. Data used in preparation of this article were obtained from the Alzheimer's Disease Neuroimaging Initiative (ADNI) database (adni.loni.usc.edu). As such, the investigators within the ADNI contributed to the design and implementation of ADNI and/ 
or provided data but did not participate in analysis or writing of this report. A complete listing of ADNI investigators can be found at: http://adni.loni.usc.edu/wp-content/uploads/how_to_apply/ADNI_ Acknowledgement_List.pdf.

\section{Compliance with ethical standards}

Conflict of interest The authors declare that they have no conflict of interest.

Publisher's note Springer Nature remains neutral with regard to jurisdictional claims in published maps and institutional affiliations.

Open Access This article is licensed under a Creative Commons Attribution 4.0 International License, which permits use, sharing, adaptation, distribution and reproduction in any medium or format, as long as you give appropriate credit to the original author(s) and the source, provide a link to the Creative Commons license, and indicate if changes were made. The images or other third party material in this article are included in the article's Creative Commons license, unless indicated otherwise in a credit line to the material. If material is not included in the article's Creative Commons license and your intended use is not permitted by statutory regulation or exceeds the permitted use, you will need to obtain permission directly from the copyright holder. To view a copy of this license, visit http://creativecommons. org/licenses/by/4.0/.

\section{References}

1. Albert MS, DeKosky ST, Dickson D, Dubois B, Feldman HH, Fox NC, et al. The diagnosis of mild cognitive impairment due to Alzheimer's disease: recommendations from the National Institute on Aging-Alzheimer's Association workgroups on diagnostic guidelines for Alzheimer's disease. Alzheimers Dement. 2011;7:270-9.

2. Dubois B, Feldman HH, Jacova C, Hampel H, Molinuevo JL, Blennow $\mathrm{K}$, et al. Advancing research diagnostic criteria for Alzheimer's disease: the IWG-2 criteria. Lancet Neurol. 2014;13:614-29.

3. McKhann GM, Knopman DS, Chertkow H, Hyman BT, Jack CR Jr., Kawas CH, et al. The diagnosis of dementia due to Alzheimer's disease: recommendations from the National Institute on AgingAlzheimer's Association workgroups on diagnostic guidelines for Alzheimer's disease. Alzheimers Dement. 2011;7:263-9.

4. Sperling RA, Aisen PS, Beckett LA, Bennett DA, Craft S, Fagan $\mathrm{AM}$, et al. Toward defining the preclinical stages of Alzheimer's disease: recommendations from the National Institute on AgingAlzheimer's Association workgroups on diagnostic guidelines for Alzheimer's disease. Alzheimers Dement. 2011;7:280-92.

5. Jack CR, Bennett DA, Blennow K, Carrillo MC, Dunn B, Haeberlein SB, et al. NIA-AA research framework: toward a biological definition of Alzheimer's disease. Alzheimers Dement. 2018;14:535-62.

6. Zwan M, van Harten A, Ossenkoppele R, Bouwman F, Teunissen $\mathrm{C}$, Adriaanse $\mathrm{S}$, et al. Concordance between cerebrospinal fluid biomarkers and [11C]PIB PET in a memory clinic cohort. J Alzheimers Dis. 2014;41:801-7.

7. Koivunen J, Pirttilä T, Kemppainen N, Aalto S, Herukka SK, Jauhianen AM, et al. PET amyloid ligand [11C]PIB uptake and cerebrospinal fluid beta-amyloid in mild cognitive impairment. Dement Geriatr Cogn Disord. 2008;26:378-83.

8. Jagust WJ, Landau SM, Shaw LM, Trojanowski JQ, Koeppe RA, Reiman EM, et al. Relationships between biomarkers in aging and dementia. Neurology. 2009;73:1193-9.
9. Fagan AM, Mintun MA, Shah AR, Aldea P, Roe CM, Mach RH, et al. Cerebrospinal fluid tau and ptau(181) increase with cortical amyloid deposition in cognitively normal individuals: implications for future clinical trials of Alzheimer's disease. EMBO Mol Med. 2009;1:371-80.

10. Cairns NJ, Ikonomovic MD, Benzinger T, Storandt M, Fagan AM, Shah AR, et al. Absence of Pittsburgh compound B detection of cerebral amyloid beta in a patient with clinical, cognitive, and cerebrospinal fluid markers of Alzheimer disease: a case report. Arch Neurol. 2009;66:1557-62.

11. Ewers M, Insel P, Jagust WJ, Shaw L, Trojanowski JQ, Aisen P, et al. CSF biomarker and PIB-PET-derived beta-amyloid signature predicts metabolic, gray matter, and cognitive changes in nondemented subjects. Cereb Cortex. 2012;22:1993-2004.

12. Schöll M, Wall A, Thordardottir S, Ferreira D, Bogdanovic N, Långström $\mathrm{B}$, et al. Low $\mathrm{PiB}$ PET retention in presence of pathologic CSF biomarkers in Arctic APP mutation carriers. Neurology. 2012;79:229-36.

13. Landau SM, Lu M, Joshi AD, Pontecorvo M, Mintun MA, Trojanowski JQ, et al. Comparing positron emission tomography imaging and cerebrospinal fluid measurements of beta-amyloid. Ann Neurol. 2013;74:826-36.

14. Palmqvist S, Zetterberg H, Blennow K, Vestberg S, Andreasson $\mathrm{U}$, Brooks DJ, et al. Accuracy of brain amyloid detection in clinical practice using cerebrospinal fluid $\beta$-amyloid 42: a crossvalidation study against amyloid positron emission tomography. JAMA Neurol. 2014;71:1282-9.

15. Mattsson N, Insel PS, Donohue M, Landau S, Jagust WJ, Shaw $\mathrm{LM}$, et al. Independent information from cerebrospinal fluid amyloid-beta and florbetapir imaging in Alzheimer's disease. Brain. 2015;138:772-83.

16. Adamczuk K, Schaeverbeke J, Vanderstichele HM, Lilja J, Nelissen N, Van Laere K, et al. Diagnostic value of cerebrospinal fluid $\mathrm{A} \beta$ ratios in preclinical Alzheimer's disease. Alzheimers Res Ther. 2015;7:75.

17. Weston PS, Paterson RW, Modat M, Burgos N, Cardoso MJ, Magdalinou N, et al. Using florbetapir positron emission tomography to explore cerebrospinal fluid cut points and gray zones in small sample sizes. Alzheimers Dement. 2015;1:440-6.

18. Leuzy A, Chiotis K, Hasselbalch SG, Rinne JO, de Mendonca A, Otto M, et al. Pittsburgh compound B imaging and cerebrospinal fluid amyloid-beta in a multicentre European memory clinic study. Brain. 2016;139:2540-53.

19. Palmqvist S, Mattsson N, Hansson O, Alzheimer's Disease Neuroimaging I. Cerebrospinal fluid analysis detects cerebral amyloidbeta accumulation earlier than positron emission tomography. Brain. 2016;139:1226-36.

20. Zwan MD, Rinne JO, Hasselbalch SG, Nordberg A, Lleó A, Herukka SK, et al. Use of amyloid-PET to determine cutpoints for CSF markers: a multicenter study. Neurology. 2016; 86:50-8.

21. Janelidze S, Zetterberg $H$, Mattsson N, Palmqvist S, Vanderstichele $\mathrm{H}$, Lindberg $\mathrm{O}$, et al. CSF A $\beta 42 / \mathrm{A} \beta 40$ and $\mathrm{A} \beta 42 / \mathrm{A} \beta 38$ ratios: better diagnostic markers of Alzheimer disease. Ann Clin Transl Neurol. 2016;3:154-65.

22. Niemantsverdriet E, Ottoy J, Somers C, De Roeck E, Struyfs H, Soetewey $F$, et al. The cerebrospinal fluid $A \beta 1-42 / A \beta 1-40$ ratio improves concordance with amyloid-PET for diagnosing Alzheimer's disease in a clinical setting. $J$ Alzheimers Dis. 2017;60:561-76.

23. Lewczuk P, Matzen A, Blennow K, Parnetti L, Molinuevo JL, Eusebi $\mathrm{P}$, et al. Cerebrospinal fluid A $\beta 42 / 40$ corresponds better than A $\beta 42$ to amyloid PET in Alzheimer's disease. J Alzheimers Dis. 2017;55:813-22.

24. Palmqvist S, Schöll M, Strandberg O, Mattsson N, Stomrud E, Zetterberg $\mathrm{H}$, et al. Earliest accumulation of $\beta$-amyloid occurs 
within the default-mode network and concurrently affects brain connectivity. Nat Commun. 2017;8:1214.

25. Álvarez I, Aguilar M, González JM, Ysamat M, Lorenzo-Bosquet C, Alonso A, et al. Clinic-based validation of cerebrospinal fluid biomarkers with Florbetapir PET for diagnosis of dementia. J Alzheimers Dis. 2018;61:135-43.

26. Doecke JD, Rembach A, Villemagne VL, Varghese S, RaineySmith S, Sarros S, et al. Concordance between cerebrospinal fluid biomarkers with Alzheimer's disease pathology between three independent assay platforms. J Alzheimers Dis. 2018;61:169-83.

27. Hansson O, Seibyl J, Stomrud E, Zetterberg H, Trojanowski JQ, Bittner T, et al. CSF biomarkers of Alzheimer's disease concord with amyloid- $\beta$ PET and predict clinical progression: a study of fully automated immunoassays in BioFINDER and ADNI cohorts. Alzheimers Dement. 2018;14:1470-81.

28. de Wilde A, Reimand J, Teunissen CE, Zwan M, Windhorst AD, Boellaard R, et al. Discordant amyloid- $\beta$ PET and CSF biomarkers and its clinical consequences. Alzheimers Res Ther. 2019;11:78.

29. Müller EG, Edwin TH, Stokke C, Navelsaker SS, Babovic A, Bogdanovic N, et al. Amyloid- $\beta$ PET-correlation with cerebrospinal fluid biomarkers and prediction of Alzheimer's disease diagnosis in a memory clinic. PLoS ONE. 2019;14:e0221365.

30. Alcolea D, Pegueroles J, Muñoz L, Camacho V, López-Mora D, Fernández-León A, et al. Agreement of amyloid PET and CSF biomarkers for Alzheimer's disease on Lumipulse. Ann Clin Transl Neurol. 2019;6:1815-24.

31. Carandini T, Arighi A, Sacchi L, Fumagalli GG, Pietroboni AM, Ghezzi L, et al. Testing the 2018 NIA-AA research framework in a retrospective large cohort of patients with cognitive impairment: from biological biomarkers to clinical syndromes. Alzheimers Res Ther. 2019;11:84.

32. Manca C, Hopes L, Kearney-Schwartz A, Roch V, Karcher G, Baumann C, et al. Assessment of $18 \mathrm{~F}$-florbetaben amyloid PET imaging in patients with suspected Alzheimer's disease and isolated increase in cerebrospinal fluid tau proteins. J Alzheimers Dis. 2019;68:1061-9.

33. Spallazzi M, Barocco F, Michelini G, Immovilli P, Taga A, Morelli N, et al. CSF biomarkers and amyloid PET: concordance and diagnostic accuracy in a MCI cohort. Acta Neurol Belg. 2019;119:445-52.

34. Ramusino MC, Garibotto V, Bacchin R, Altomare D, Dodich A, Assal $\mathrm{F}$, et al. Incremental value of amyloid-PET versus CSF in the diagnosis of Alzheimer's disease. Eur J Nucl Med Mol Imaging. 2020;47:270-80.

35. Toledo JB, Bjerke M, Da X, Landau SM, Foster NL, Jagust W, et al. Nonlinear association between cerebrospinal fluid and Florbetapir F-18 beta-amyloid measures across the spectrum of Alzheimer disease. JAMA Neurol. 2015;72:571-81.

36. Cohen AD, Landau SM, Snitz BE, Klunk WE, Blennow K, Zetterberg H. Fluid and PET biomarkers for amyloid pathology in Alzheimer's disease. Mol Cell Neurosci. 2019;97:3-17.

37. Ikonomovic MD, Klunk WE, Abrahamson EE, Mathis CA, Price JC, Tsopelas ND, et al. Post-mortem correlates of in vivo PiBPET amyloid imaging in a typical case of Alzheimer's disease. Brain. 2008;131:1630-45.

38. Lowe VJ, Lundt ES, Albertson SM, Przybelski SA, Senjem ML, Parisi JE, et al. Neuroimaging correlates with neuropathologic schemes in neurodegenerative disease. Alzheimers Dement. 2019;15:927-39.
39. Murray ME, Lowe VJ, Graff-Radford NR, Liesinger AM, Cannon A, Przybelski SA, et al. Clinicopathologic and 11C-Pittsburgh compound B implications of Thal amyloid phase across the Alzheimer's disease spectrum. Brain. 2015;138:1370-81.

40. Clark CM, Pontecorvo MJ, Beach TG, Bedell BJ, Coleman RE, Doraiswamy PM, et al. Cerebral PET with florbetapir compared with neuropathology at autopsy for detection of neuritic amyloidbeta plaques: a prospective cohort study. Lancet Neurol. 2012;11:669-78.

41. Olsson A, Vanderstichele H, Andreasen N, De Meyer G, Wallin A, Holmberg B, et al. Simultaneous measurement of beta-amyloid (1-42), total tau, and phosphorylated tau (Thr181) in cerebrospinal fluid by the xMAP technology. Clin Chem. 2005;51:336-45.

42. Landau S, Jagust WJ. Florbetapir processing methods. Caliornia: Helen Wills Neuroscience Institute, UC Berkeley and Lawrence Berkeley National Laboratory; 2015. http://ida.loni.usc.edu.

43. Landau SM, Mintun MA, Joshi AD, Koeppe RA, Petersen RC, Aisen PS, et al. Amyloid deposition, hypometabolism, and longitudinal cognitive decline. Ann Neurol. 2012;72:578-86.

44. Jack CR, Bernstein MA, Fox NC, Thompson P, Alexander G, Harvey D, et al. The Alzheimer's disease neuroimaging initiative (ADNI): MRI methods. J Magn Reson Imaging. 2008;27:685-91.

45. DeCarli C, Carmichael O, He J. MRI infarct assessment in ADNI. Davis: Department of Neurology and Center for Neuroscience, University of California; 2013.

46. DeCarli C, Maillard P, Fletcher E. Four tissue segmentation in ADNI II. Davis: Department of Neurology and Center for Neuroscience, University of California; 2013.

47. Desikan RS, Fan CC, Wang Y, Schork AJ, Cabral HJ, Cupples LA, et al. Genetic assessment of age-associated Alzheimer disease risk: Development and validation of a polygenic hazard score. PLoS Med. 2017;14:e1002258.

48. Shaw LM, Vanderstichele H, Knapik-Czajka M, Clark CM, Aisen PS, Petersen RC, et al. Cerebrospinal fluid biomarker signature in Alzheimer's disease neuroimaging initiative subjects. Ann Neurol. 2009;65:403-13.

49. De Meyer G, Shapiro F, Vanderstichele H, Vanmechelen E, Engelborghs S, De Deyn PP, et al. Diagnosis-independent Alzheimer disease biomarker signature in cognitively normal elderly people. Arch Neurol. 2010;67:949-56.

50. Joshi AD, Pontecorvo MJ, Clark CM, Carpenter AP, Jennings DL, Sadowsky $\mathrm{CH}$, et al. Performance characteristics of amyloid PET with florbetapir F 18 in patients with alzheimer's disease and cognitively normal subjects. J Nucl Med. 2012;53:378-84.

51. Clark CM, Schneider JA, Bedell BJ, Beach TG, Bilker WB, Mintun MA, et al. Use of florbetapir-PET for imaging betaamyloid pathology. JAMA. 2011;305:275-83.

52. Ott BR, Cohen RA, Gongvatana A, Okonkwo OC, Johanson CE, Stopa EG, et al. Brain ventricular volume and cerebrospinal fluid biomarkers of Alzheimer's disease. J Alzheimers Dis. 2010;20:647-57.

53. Lucey BP, Hicks TJ, McLeland JS, Toedebusch CD, Boyd J, Elbert DL, et al. Effect of sleep on overnight cerebrospinal fluid amyloid $\beta$ kinetics. Ann Neurol. 2018;83:197-204.

54. Lim YY, Mormino EC. Initiative AsDN. APOE genotype and early $\beta$-amyloid accumulation in older adults without dementia. Neurology. 2017;89:1028-34. 